\title{
Research Article WLAN Technologies for Audio Delivery
}

\author{
Nicolas-Alexander Tatlas, ${ }^{1}$ Andreas Floros, ${ }^{2}$ Thomas Zarouchas, ${ }^{1}$ and John Mourjopoulos ${ }^{1}$ \\ ${ }^{1}$ Audio Technology Group, Department of Electrical and Computer Engineering, University of Patras, 26500 Patras, Greece \\ ${ }^{2}$ Department of Audio Visual Arts, Ionian University, Plateia Tsirigoti 7, 49100 Corfu, Greece
}

Received 21 April 2007; Revised 30 August 2007; Accepted 27 December 2007

Recommended by Tasos Dagiuklas

\begin{abstract}
Audio delivery and reproduction for home or professional applications may greatly benefit from the adoption of digital wireless local area network (WLAN) technologies. The most challenging aspect of such integration relates the synchronized and robust real-time streaming of multiple audio channels to multipoint receivers, for example, wireless active speakers. Here, it is shown that current WLAN solutions are susceptible to transmission errors. A detailed study of the IEEE802.11e protocol (currently under ratification) is also presented and all relevant distortions are assessed via an analytical and experimental methodology. A novel synchronization scheme is also introduced, allowing optimized playback for multiple receivers. The perceptual audio performance is assessed for both stereo and 5-channel applications based on either PCM or compressed audio signals.
\end{abstract}

Copyright (C) 2007 Nicolas-Alexander Tatlas et al. This is an open access article distributed under the Creative Commons Attribution License, which permits unrestricted use, distribution, and reproduction in any medium, provided the original work is properly cited.

\section{INTRODUCTION}

In typical home and professional applications, digital audio can be delivered from any source to single or multiple receivers, through local area networks (LANs). Thus, the interconnection between devices may be simplified, and the communication between audio sources, receivers, and other multimedia devices may be optimized [1]. Additionally, in this case, an Internet connection could transparently be considered as an additional source with enhanced features, such as audio on demand. A further improvement would be the employment of wireless local area networks (WLANs). The first obvious practical benefit of using a WLAN is that interconnection cables are eliminated and, depending on the application, a number of wireless transceivers (access points-APs and wireless Stations-STAs) can be installed and appropriately configured for realizing any required audio delivery scenario. An additional advantage is that the same WLAN infrastructure can also service data transmissions between personal computers and other digital devices; hence, such systems will be compatible with a wide range of applications and eventually will present extremely flexible and cost-effective alternative to the present home entertainment chain.

A number of wireless audio products already exist in the market, starting from analog systems operating in the area of 800-900 MHz (e.g., wireless microphones, in-ear monitors, and loudspeakers) up to proprietary wireless digital stream- ing technologies. In such systems, the wireless transmission protocol is application specific for reducing the implementation complexity and cost. This restricts equipment compatibility and raises interoperability issues between different manufacturing designs, often to the extent that the concept of networking is defied. To overcome such compatibility issues, established wireless networking standards should be employed, such as Bluetooth [2], HyperLAN/2 [3], HomeRF [4], and the IEEE 802.11 family of protocols [5]. Among them, the latter specification currently represents the most promising scheme for wireless audio applications, due to its wide adoption and the continuous ratification process which will provide significant enhancements in many state-of-theart networking aspects, such as security and adaptive topology control.

Despite the recent advances on transmission rates (the $802.11 \mathrm{~g}$ specification [6] offers a theoretical maximum of $54 \mathrm{Mbps}$, while the upcoming $802.11 \mathrm{n}$ draft extends this rate up to $270 \mathrm{Mbps}$ ), the existing "best-effort" nature of WLAN protocols introduces practical limits for real-time audio open-air streaming. To overcome such constraints, the transmission protocol must provide quality-of-service (QoS) guarantees [7]. Additionally, although QoS represents the major requirement for point-to-point multimedia streaming applications, the development of wireless multichannel audio products (e.g., for home theater) raises the challenging issue of synchronization between the wireless receivers. 
A number of techniques for network time synchronization have been proposed for audio over IP networks [1]. Generally, there are two such synchronization issues: (a) local clock synchronization problems due to a (variable) offset between the hardware clocks of the remote receivers, and (b) intrastream (packet ordering and timing in one stream) and interstream (temporal relationship among different streams) synchronization, both defined in [8]. Considering a two-channel system, usually local clock synchronization issues may be manifested as audio pitch modification, change of source directional perception, or even audible noise for rapidly changing clock offset [9]. Additionally, loss of intrastream synchronization may be perceived as gaps and phase mismatches during reproduction, while interstream synchronization may lead to two channels being perceived as separate sources and shifts in the acoustic image spatial position.

A previous published study [9] has shown that local clock synchronization can be efficiently achieved in the application layer using the available hardware, leading to a maximum clock drift of less than $0.1 \mathrm{~ms}$. On the other hand, interstream synchronization of high-quality multichannel audio still represents a very challenging research field, since the methods already presented mainly focus on intrastream synchronization for single-channel voice applications or interstream synchronization for point-to-point multichannel applications [10].

The aim of this study is to give an in-depth overview of all the issues related to audio WLAN delivery, focusing on two areas: (i) the effect of the wireless environment on the overall audio playback quality and (ii) the issue of interstream synchronization for real-time playback of high quality, (uncompressed and compressed) audio multichannel streams over a WLAN platform. The wireless transmission protocol considered in this work is the well-established $802.11 \mathrm{~b}$ standard with the QoS enhancements defined in the latest version of the IEEE802.11e draft amendment [11]. Additionally, a novel interstream synchronization technique is introduced for synchronizing discrete audio channel playback among a number of wireless loudspeakers, using typical, off-the-self, transceiver hardware. In this way, the study is not bounded by product-specific protocols and implementations.

The rest of the paper is organized as follows. Section 2 provides a general background on wireless networking and QoS for audio applications. The architecture of the system employed and a theoretical timing and distortion playback analysis is presented in Section 3. In the same Section, a novel method (termed as Consistent Delay SynchronizationCoDeS) is introduced, which compensates for any playback distortions introduced by any network interchannel variable delay. Section 4 presents the test methods employed and examines the timing error results and the audibility for the various tests described. Finally, the conclusions of this work are summarized in Section 5.

\section{WIRELESS NETWORKS FOR AUDIO APPLICATIONS}

There are two classes of audio applications that can be supported by a WLAN.

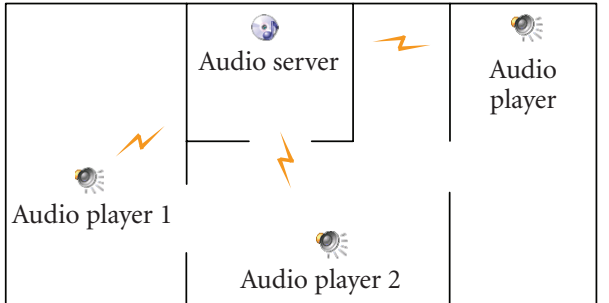

(a)

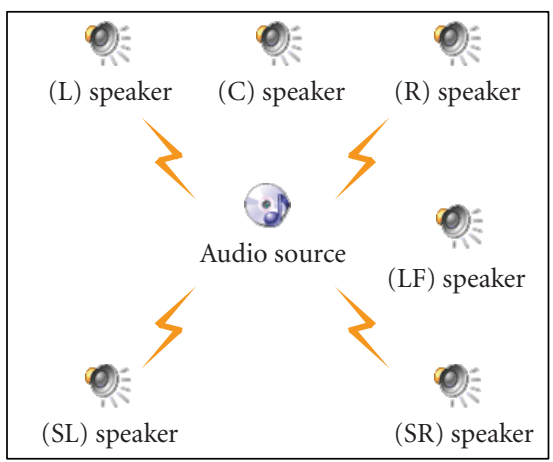

(b)

FIgUre 1: Typical WLAN digital audio systems: (a) simple pointto-point wireless audio delivery, (b) wireless multichannel playback setup.

(a) Simple point-to-point home audio delivery (see Figure 1(a)) where an audio server wirelessly transmits in real time the same or different audio streams to a number of wireless audio players/receivers. No synchronization between the wireless receivers is necessary, while the maximum allowed number of remote players is dynamically adjusted by the QoS bandwidth reservation algorithms. Using the above setup, any certified WLAN-enabled audio device (including portable audio playback, laptop computers, and consumer electronics equipment) can be directly connected to the network and receive audio data on user demand. Currently, a number of products operating in the $\mathrm{S}$ Band ISM (2.40-2.48 GHz, available worldwide) and in the C-Band ISM (5.725-5.875 GHz, available in some countries) exist, including Bluetooth audio applications [12], complete wireless home networking setups $[13,14]$, as well as wireless headphone solutions [15]. Moreover, integrated home theater systems employing wireless surround channel reproduction via a single point-to-point link have been recently introduced in the consumer electronics market [16]. However, most of these systems employ compressed quality or even analog audio and are based on proprietary transmission protocols and technologies, and hence are incompatible to the emerging WLAN standards.

(b) Wireless point-to-multiple receivers (see Figure 1(b)), where typically 6 loudspeakers of a 5.1 channel home theater setup can be wirelessly connected to an audio source. In this case, the digital audio source transmits audio data to the appropriate wireless loudspeaker which should perform 
simultaneous and synchronized (relative to all other receivers) playback in real time. Hence, local (i.e., hardware) clock as well as packet playout synchronization methods are required for eliminating unpredicted channel shifts and phase distortions. Additionally, in this case, the pre- and power amplification modules can be assumed to be integrated within the loudspeaker and it is likely that digital audio amplifiers can be employed for greater power efficiency, better system integration, as well as reduced size and cost [17].

A WLAN-based multichannel audio system will additionally benefit from high-level procedures for automatic receiver position discovery. These will be able to take into consideration lower protocol layer metrics currently being defined by the $802.11 \mathrm{k}$ enhancement in order to allow each wireless loudspeaker to define its' function (e.g., left, right channel, etc.) within the multichannel setup. Moreover, application-layer mechanisms must be also supported for allowing the user to control several playback parameters, such as relative channel volume, delay, and so on. All these aspects represent challenging topics which may allow in the near future the replacement of typical wired speakers with novel WLAN-enabled active speakers.

\subsection{Quality-of-service over WLANs}

As it was previously mentioned, the accepted term for providing time-critical services over a network is quality of service (QoS), which refers to the capability of a network to constantly provide specific service guarantees. An introduction to QoS issues for streaming audio over WLANs can be found in [18], where a very early version of the $802.11 \mathrm{e}$ draft amendment is described. However, during a long ratification process, additional QoS characteristics were introduced and are briefly described here. The current 802.11 e specification [11] defines two access types. Using "priority differentiation," all transmitting devices contend for the wireless medium under the rules defined by the enhanced distributed channel access (EDCA). On the other hand, when "resource reservation" is used, all wireless transmissions are centrally controlled, following the so-called hybrid controlled channel access (HCCA) rules.

A previous work [19] has defined the minimum transmission requirements for wirelessly distributing CD-quality and multichannel audio using EDCA. In this work, the HCCA mechanism is considered which, to the best of the author's knowledge, is not yet exploited for high-quality audio applications, while, according to [20], it achieves QoS performance for real-time multimedia traffic. A detailed description of HCCA is out of the scope of this work and can be found in [21]. Briefly, under HCCA, an STA transmits only upon the reception of a polling frame sent by the AP. The allowed transmission time lengths are calculated using a number of traffic specifications (TSPECs) declared by the STAs upon their service initialization. Typical TSPEC parameters are the traffic mean/maximum data rate, the corresponding packet length, and the physical (PHY) transmission rate. $802.11 \mathrm{e}$ additionally includes the simple scheduler (SiS) description for defining the minimum require- ments of any HCCA service scheduler. An alternative scheduler [22] is also considered here, termed scheduling based on estimated transmission times-earliest due date (SETTEDD), which aims to improve QoS performance under variable transmission conditions.

\subsection{Wireless multichannel audio system topology}

The general architecture of the wireless multichannel system considered here is shown in Figure 2 and consists of (a) one digital audio source integrated with a wireless QoS AP transceiver forming a wireless digital audio source (WiDAS). Appropriate buffering stages are also employed in order to transform the digital audio samples stream into packets of appropriate length. (b) A number $(M \geq 2)$ of wireless digital audio receivers (WiDARs). Typically, these systems can be considered as wireless self-powered loudspeakers, containing a wireless subsystem which recreates the audio stream from the received packets.

Briefly, the basic functionality of the above setup is as follows. The WiDAS transmits the digital audio and control information to the WiDARs. In case of linear PCM-coded audio, each audio channel is transmitted to the appropriate WiDAR, based on the identification information provided by the topology detection procedure. For compressed-quality audio, it is likely that all the audio channels will be multiplexed in a single digital stream. Hence, the WiDAS can broadcast the audio information to all WiDARs, each being responsible for decoding the transmitted stream and for selecting the appropriate audio channel for playback. However, the lack of an adaptive 802.11 medium access control (MAC) layer retransmission mechanism when broadcasting, renders the overall transmission quality inadequate. Thus, unicast transmissions are generally preferred.

The WiDAS controls all wireless transmissions and attempts to sustain the appropriate mean data rate necessary for real-time audio reproduction. However, due to the stochastic link conditions, the instantaneous throughput and transmission delay values may significantly differ. As human hearing is highly delay sensitive, prebuffering mechanisms must be employed [23]. Long buffering queues can better compensate for variable channel conditions; however, there is a trade-off between the perceived user interaction latency and the buffering length. These restrictions force the buffering stages to be of predetermined and finite size, which, as it will be explained in the next section, may result in audible distortions during real-time playback.

\section{WIRELESS TRANSMISSION TIMING ANALYSIS}

To study the wireless packet-oriented transmission of digital audio streams and any possible errors induced, a sourceto-receiver timing analysis will be now introduced. Initially, assuming uncompressed audio PCM streams, the WLAN audio source produces audio samples at a constant rate of $N f_{s}$ (bytes/s), where $N$ is the byte resolution per sample and $f_{s}$ $(\mathrm{Hz})$ is the sampling rate. The transmitter software assembles 


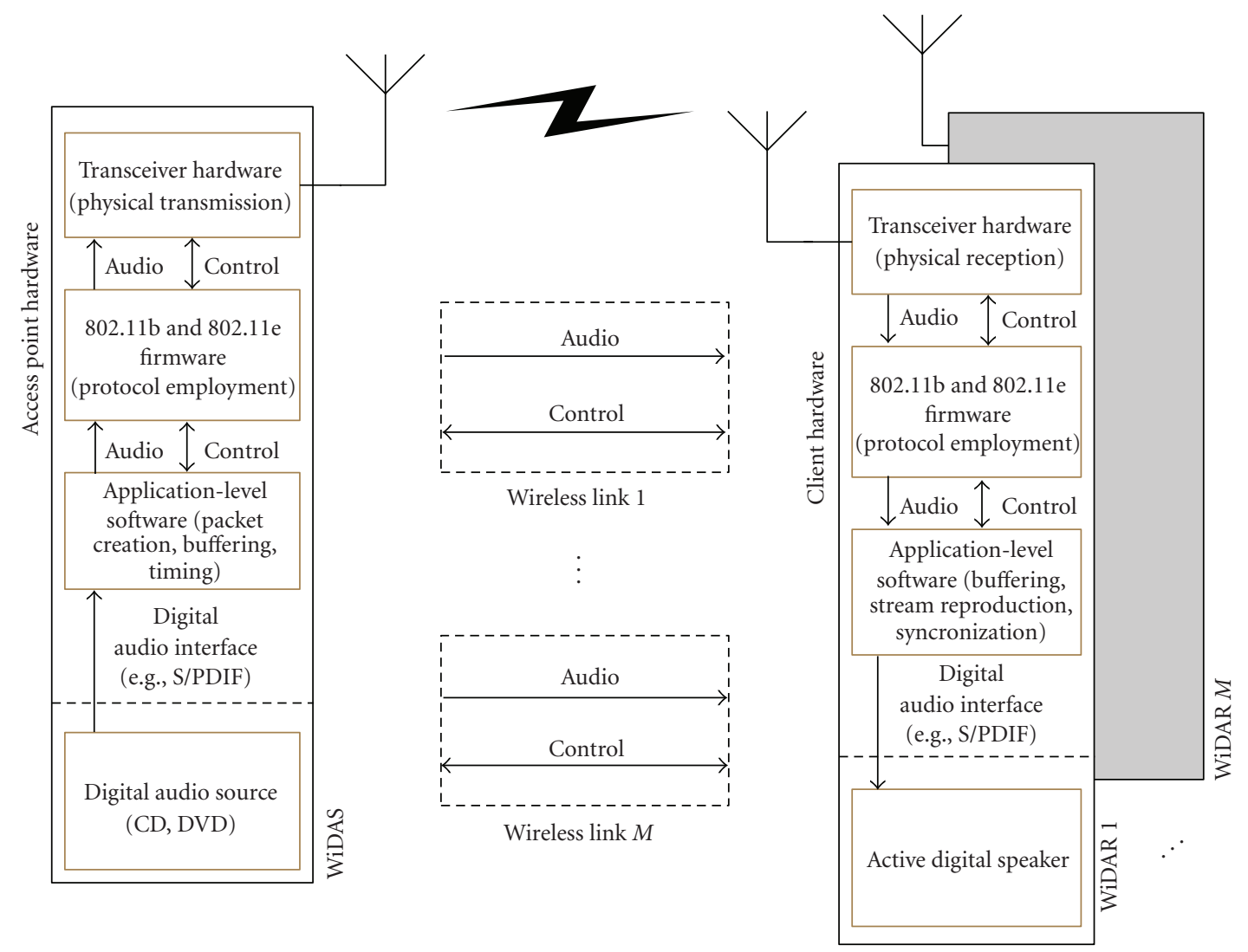

FIGURE 2: Architecture of the WLAN multichannel playback system considered in the study.

appropriate number of samples, to form packets of length $L_{p}$ (bytes). Thus, each packet is generated every

$$
T_{g}=\frac{L_{p}}{N f s}(s)
$$

For compressed audio, such as MPEG-1 Layer III streams, the audio source produces data at predefined intervals of

$$
T_{g}=\frac{L_{p}}{b}(s)
$$

where $\mathrm{L}_{p}$ is the frame length in bytes and $b$ is the predetermined compressed audio total bitrate (bps). Hence in both cases, the ith packet generation time is quantized in multiples of $T_{g}$.

\subsection{Wireless audio transmission}

Upon generation of each audio packet, the packet is inserted into the WiDAS transmission (Tx) buffer at instances $t_{g}(i)$. However, assuming that no upper-layer recovery mechanism is employed, if the buffer is full, then the packet will be dropped. This is statistically equivalent to applying packet-aging functions during transmissions, meaning that each packet in the queue will be deleted if it is not successfully transmitted in a predefined time interval. Thus, buffer overflows caused by transmission errors lead to permanent packet losses. It should be also noted that, despite the lossy wireless transmission, out-of-order packet arrival cannot occur, as no alternative routing nodes exist. Hence no packet reordering mechanisms are necessary, and a buffered packet will be transmitted after the transmission of all the previously queued packets. Assuming that $d_{\text {Txbuffer }}(i)$ is the time delay between the packet insertion in the Tx queue and its transmission to the receiver, then the transmission time instance $t_{\mathrm{Tx}}(i)$ for the $i$ th packet will be equal to

$$
t_{\mathrm{Tx}}(i)=t_{\mathrm{g}}(i)+d_{\mathrm{Txbuffer}}(i) .
$$

Furthermore, the delay $d_{\text {Txbuffer }}(i)$ between the packet insertion in the Tx queue and its successful transmission depends on the number of packets already in queue, and hence the actual transmission delay of a given packet will be $d_{\mathrm{Tx}}(i)$, as shown in Figure 3,

$$
d_{\text {Txbuffer }}(i)=d_{\text {Txbuffer }}(j)-(i-j) T_{\mathrm{g}}+d_{\mathrm{Tx}}(i),
$$

where $j$ is the index of the immediately preceding packet successfully inserted in the Tx queue.

$d_{\mathrm{Tx}}(i)$ represents the time interval elapsed between the movement of the $i$ th packet to the first position of the $\mathrm{Tx}$ queue and its successful transmission. This delay depends on the subsequent polling of the corresponding stream, resulting into successful packet transmissions, depending on the transmission physical rate, transmission time overheads, retransmission delays, and the scheduler type. Here, the propagation delay between the transmission and the reception 


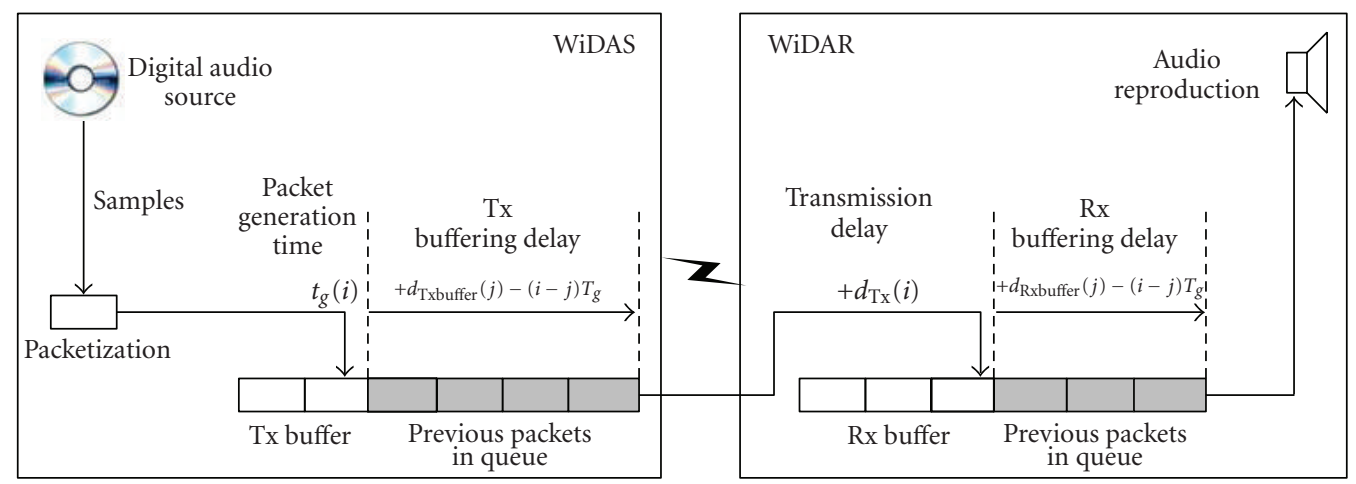

FIGURE 3: Representation of delay generation in packet-oriented WLAN audio transmission.

point will be considered to be negligible compared to such delays (e.g., retransmission, etc.), something that is largely true especially for high physical rates.

\subsection{Wireless audio reception}

Upon reception, each packet will be placed in the reception $(\mathrm{Rx})$ buffer, as long as there is sufficient space, at instances $t_{\mathrm{Rx}}(i)$ equal to $t_{\mathrm{Tx}}(i)$. If the Rx buffer is full, then this incoming packet will be permanently disregarded. Each buffered packet will be successfully reproduced provided that all previous packets successfully inserted in the Rx queue have been reproduced. Then, the time instance for the $i$ th packet reproduction is equal to

$$
t_{p}(i)=t_{\mathrm{Rx}}(i)+d_{\mathrm{Rxbuffer}}(i),
$$

where $d_{\text {Txbuffer }}(i)$ is the delay between the packet insertion in the queue and its reproduction (also shown in Figure 3)

$$
d_{\text {Rxbuffer }}(i)=d_{\text {Rxbuffer }}(j)-(i-j) T_{\mathrm{g}}+T_{\mathrm{g}},
$$

where $j$ is the index of the last packet in the queue before the insertion of the $i$ th packet.

To denote the successful packet insertion, either in the Tx or Rx queue, a function $\delta_{s}(i)$ can be defined such that

$$
\delta_{s}(i)= \begin{cases}0, & \text { if either queue is full } \\ 1, & \text { otherwise }\end{cases}
$$

Using the above equations, the packet reproduction time $t_{p}(i)$ can be defined as

$$
t_{p}(i)=\delta_{\mathrm{s}}(i)\left[t_{\mathrm{g}}(i)+d(i)\right]
$$

where

$$
d(i)=d_{\text {Txbuffer }}(i)+d_{\text {Rxbuffer }}(i)
$$

is the total delay induced by the WLAN between packet generation and final playback. Packet losses will cause the playout delay of succeeding packets to fluctuate, since the corresponding $t_{\text {Txbuffer }}$ and $t_{\text {Rxbuffer }}$ values will appropriately change, as will be described below.
If playback prebuffering is employed, then the first packet reproduction will be delayed in the reception queue for a predefined amount of time, $t_{\text {prebuffering, }}$ causing the first packet received to be delayed by this time so that

$$
d(k)=t_{\text {prebuffering }},
$$

where $k$ is the first packet successfully inserted into the reproduction queue; typically, $k=1$. It is obvious that this delay propagates to all subsequent packets.

\subsection{Time-related network distortions}

If a packet with index $i$ is dropped from either the Tx or $\mathrm{Rx}$ queue $\left(\delta_{s}(i)=0\right)$, a discontinuity in the reproduction will occur, which may be audible during audio playback. Moreover, in the case of a stereo or multichannel audio setup, this would additionally cause loss of channel synchronization with at least one channel leading, unless a compensation strategy is employed in the system, such as inserting an empty packet in the queue. As will be shown in Section 4, this condition can appear under heavy network load. In practice, for a given PHY transmission rate, excessive MAC layer retransmissions may occur and/or, for the case in which the transmitting application is attempting to compensate for previous packet losses through retransmissions, the overall required channel data rate will increase. If the reproduction has actually been halted when receiving the packet, that is,

$$
t_{p}(i)-t_{p}(j)>T_{\mathrm{g}}
$$

where $j$ is the previously reproduced packet, then another kind of audible distortion will take place, since playback interrupts (silence gaps) will be introduced. In this case, at least one channel will be lagging, unless the other receivers momentarily stop playing audio data as well. For a given packet, the above two distortions can be combined, by writing

$$
t_{p}(i)-t_{p}(j)>T_{\mathrm{g}} \quad j \neq i-1 .
$$

In this case, depending on the value $(i-j-1)$ giving the packets lost and the value $\left(t_{p}(i)-t_{p}(j)-T_{g}\right)$ giving the time elapsed from stopping the playback, the receiver will be either leading or lagging, or even in phase with the other receivers. 


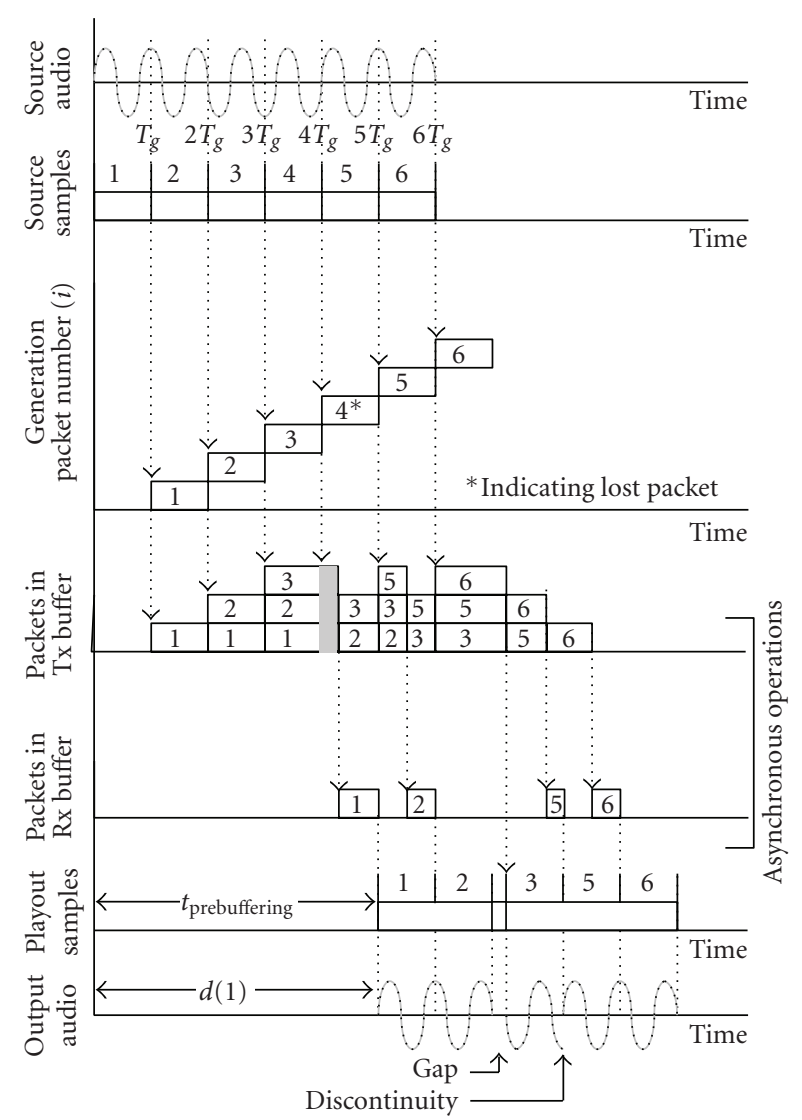

FIGURE 4: Example of timing analysis in packet-oriented digital audio WLAN transmission.

In order to assess the total amount of data excessively delayed, the concept of delayed throughput is introduced. In general, throughput is described as the sum of data successfully sent by a source over the transmission time. According to the $802.11 \mathrm{e}$, a transmission delay bound must be defined for any traffic source. Hence, the amount of data arriving after this bound, over their transmission time, is termed here as delayed throughput ( $T_{D}$, measured in Mbps) and defined to be equal to

$$
T_{D}=\frac{1}{I_{\text {total }}} \sum_{i=1}^{I_{\text {total }}} \frac{L_{p}}{d_{\mathrm{Tx}}(i)} \quad \text { for } d_{\mathrm{Tx}}(i)>\max \mathrm{SI}
$$

where $I_{\text {total }}$ is the total number of packets transmitted and $\max S I$ is the maximum service interval, defined in [11]. In case that $T_{D} \neq 0$, reproduction distortion will be introduced (i.e., relative channel delay, discontinuities, and silence gaps).

An example for reproduction gaps and discontinuities due to the wireless transmission is shown in Figure 4. Here, for illustrative purposes, the Tx and Rx buffer size is set to 3 packets. As shown, playback commences after the predetermined prebuffering time $t_{\text {prebuffering, }}$, and while packets \#1 and $\# 2$ are accurately reproduced, excessive transmission delay for packets \#1, \#2, and \#3 causes a gap in reproduction. Moreover, packet \#4 is disregarded because at the time it is pushed in the transmitter buffer, the Tx Buffer is full, causing a discontinuity in audio reproduction. Note that the trans- mission delay is not taken under consideration, thus a received packet is available for reproduction on the instance it is extracted from the Tx buffer and inserted in the Rx buffer.

\subsection{Synchronization strategy}

The preceding analysis indicates that in the case audio data packets are lost or excessively delayed, an application-level compensation strategy is necessary in order to ensure synchronized reproduction of all receivers at any given time, even if prebuffering is employed. The algorithm should have low signaling complexity (expressed in terms of additional packet exchange required for achieving synchronization) in order to ensure that no substantial network overhead is induced. As already mentioned, local (hardware) clock synchronization is not addressed here, since it has been shown to be efficiently achieved in the application layer using the available wireless transmission hardware [9].

The consistent delay synchronization (CoDeS) strategy proposed here is based on the adjustment of the packet delay so it remains consistent and independent of networking parameters and conditions. Ideally, when prebuffering is employed, the playout delay for each packet should be constant and equal to $t_{\text {prebuffering }}$. The following paragraphs explain how the CoDeS strategy compensates for variable delays utilizing information from each packet header, for the case of (a) buffer overflow and (b) buffer underflow. 


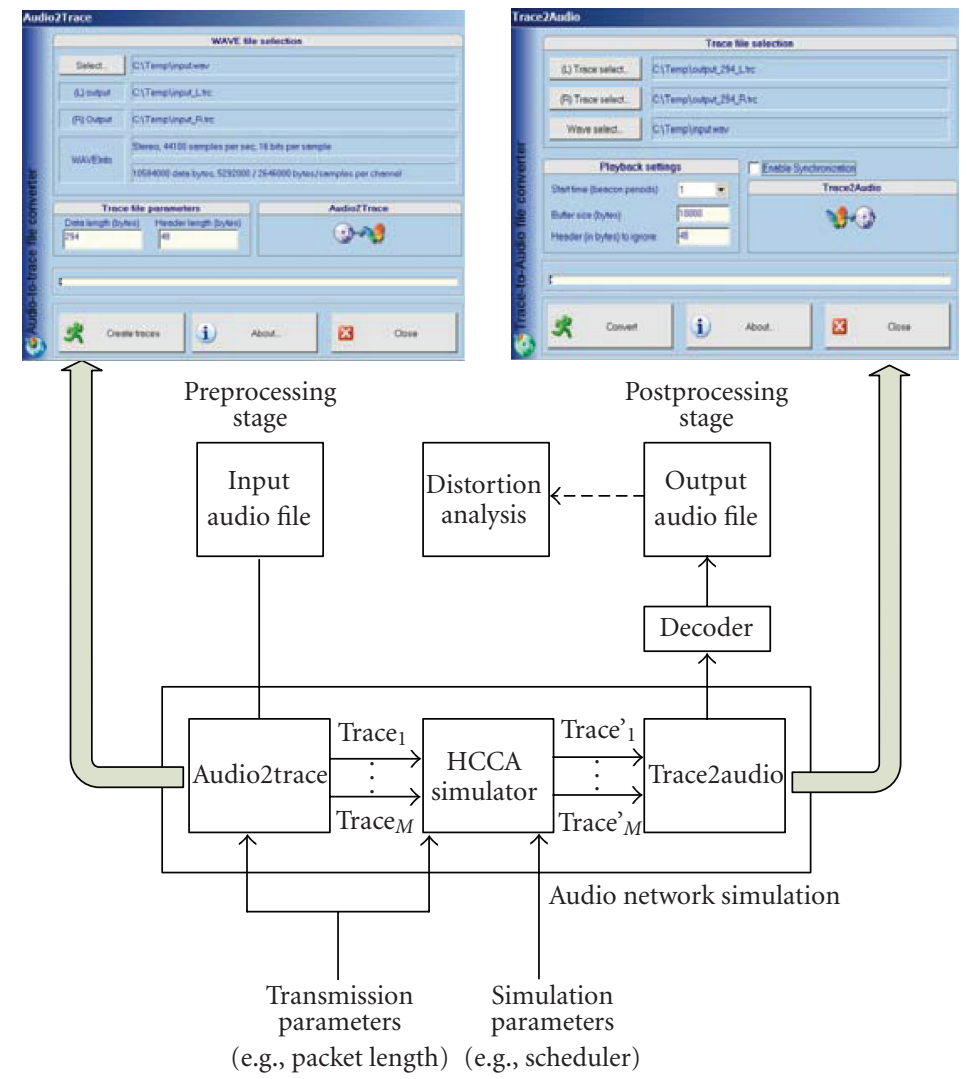

FIgURE 5: Block diagram of the WLAN audio delivery simulation.

(a) If any packet drop occurs due to buffer overflows, then the delay of the next packet in the reception queue will be reduced, that is,

$$
d(i)<t_{\text {prebuffering }}
$$

The corresponding lost packet playback time must be then compensated for, by adjusting accordingly $d_{\text {Rxbuffer }}(i)$ to $d_{\text {Rxbuffer }}^{\prime}(i)$ ensuring synchronized reproduction of all subsequent packets, that is,

$$
d_{\text {Rxbuffer }}^{\prime}(i)=d_{\text {Rxbuffer }}(i)+d(i)-t_{\text {prebuffering }} \text {. }
$$

(b) If excessive delay is introduced in the transmission path causing buffer underflow, then a packet might be reproduced after its predetermined playback time. In this case, the packet has to be disregarded. Thus, if

$$
d(i)>t_{\text {prebuffering }}
$$

then, it must be $\delta_{s}(i)=0$.

After adjusting the delays according to the above equations, the receiver may employ digital audio editing signal processing for error concealment, such as proposed in $[24,25]$, in order to ensure acceptable signal continuity.

\section{TEST METHODOLOGY AND RESULTS}

In order to realize the complete real-time wireless transmission and playback process and to evaluate any playback distortions, a computer-based test methodology was developed [26], which is illustrated in Figure 5. It consists of three main subsystems: (a) the simulation preprocessing stage, which converts the digital audio data into inputs to the HCCA simulator, (b) the HCCA simulator [27], conforming to the mandatory HCCA functionality defined in the latest $802.11 \mathrm{e}$ draft specification [11], and (c) a simulation postprocessing stage which processes the simulator's output and produces a new file containing a "reproduced" PCM version of the original digital audio data. The proposed CoDeS synchronization algorithm is also incorporated in the postprocessing subsystem to derive the "synchronized" version of the received data.

The reproduced audio file can be used for evaluating distortions for each receiver, by comparing the reproduced data to the corresponding original input waveform, as well as for evaluating possible synchronization loss between these data. The methodology described here for stereo (compressed and uncompressed) digital audio data can be easily extended to more channels (e.g., 6 audio channels as used in the DVD format). A detailed description of the functionality of the three subsystems is provided in the following paragraphs. 


\subsection{Simulation parameters}

The HCCA simulator (see Figure 5) uses trace files for external traffic modeling of any required traffic flow. A trace file describes a traffic stream in terms of the resulting packets as a function of time, a technique used in the past to model variable video traffic [27]. In this work, although the audio data rate is always constant (for either compressed or linear PCM), trace file modeling was employed for mapping the transmitted data packets to specific segments of typical audio files.

The mapping of the original audio file to trace files was performed using an application developed by the authors of [26] called Audio2Trace, as shown in Figure 5. For the case of uncompressed (PCM) audio transmission, the conversion parameters include the total duration, the audio channel bitrate, the packet header length (see below), and the userdefined pure audio data packet length $L_{p}$ (in bytes) which was set equal to 294 and 882 bytes during this work. Note that the above packet length selection was implied by the requirement of deriving a transmission schedule in whole submultiples of the beacon interval. On the other hand, for compressed transmission (e.g., MPEG-1 Layer III (mp3)), the conversion parameters are all extracted from the input file header, with the pure audio data packet length being equal to the MPEG frame length. In both cases, the UDP transport protocol was employed, which adds an 8 byte header on all transmitted packets [10], while 40 additional header bytes are reserved for future control purposes (e.g., RTP encapsulation). Taking into account the derived data bitrates for stereo linear PCM signal (1.4 Mbps for uncompressed CD-quality audio), the legacy, low-cost IEEE802.11b protocol was selected with a PHY rate equal to $11 \mathrm{Mbps}$, while a custom retransmission scheme is employed in the MAC layer. However, as explained in Section 3, packet losses may occur due to buffer overflow.

The wireless channel models employed during the simulations were obtained through measurements of real-world 802.11b-based transmission patterns in a controlled environment, with the wireless stations being at $2 \mathrm{~m}$ distance, under no interference (also referred as good channel) and medium interference (also referred as medium channel) induced by two neighboring stations in the $2.4 \mathrm{GHz}$ frequency band. More specifically, the number of the total transmissions (including retransmissions) and the total transmissions that resulted into successful delivery were measured within every beacon period and the successful delivery probability was calculated as a function of time (expressed in multiples of the beacon period). A channel model plug-in for the HCCA simulator was finally implemented, that takes into account the resulting probability values and applies them to the transmissions taking place within each simulated HCCA TXOP.

The HCCA simulator models the WiDAS and WiDAR 802.11 MAC layer functionality, assuming that the WiDAS device has a 5 Kbyte prebuffering stage, typically used in such applications, for every serviced audio stream. The simulator produces one output trace file per serviced traffic (audio channel) stream containing information for all the corresponding data packets sent, such as packet transmission con- firmation as well as the packet delay induced. This information is used by the simulation postprocessing stage (implemented by the Trace2Audio application) for deriving a new wave file representing the received (playback) version of the source audio data.

Using the Trace2Audio application, the receiver buffering stage is implemented using a first-in first-out (FIFO) reception queue with user-defined length. This queue is gradually filled with the successfully received data packets and is getting empty in a sample-by-sample basis, as the audio samples are read at a rate equal to the original PCM sampling frequency $f_{s}(\mathrm{~Hz})$. If the $\mathrm{Rx}$ queue is empty, then the output sample values are set to zero. Furthermore, a user-selectable initial latency for prebuffering purposes has been considered, in order to decrease the audible consequences of jitter in packet arrival. This latency was set in multiples of $100 \mathrm{~ms}$, which is the beacon transmission period defined by the legacy IEEE 802.11 specification. For the test cases considered here, the Rx queue length was always equal to 10000 bytes and the initial latency was set equal to 1 beacon interval (100 ms).

In the case of compressed audio transmission, an external decoder was employed in the final post processing stage. The application initially discriminates between correctly received frames and erroneous data caused by excessive delays. The decoder is used to process the correctly received frames and decode them to PCM samples, while erroneous or missing data are directly mapped to zero-value PCM digital audio samples, depending on the initial encoding bitrate and sampling rate.

The proposed CoDeS synchronization scheme described in Section 3, in practice, requires only metadata information for the packets, included within each header and timing information from the WiDAR. CoDeS has been included as an option in the Trace2Audio application modifying (when necessary) the Rx queue contents.

\subsection{Network-induced distortion evaluation}

\subsubsection{End-to-end playback delay}

In order to detect the end-to-end delay for each audio channel under all possible parameters for PCM transmission, a periodic audio test signal was selected as the system input. By comparing the original input signal to the wirelessly reproduced version, the end-to-end delay can be estimated as seen in the following figures.

Figure 6 shows typical Tx and the Rx usage for a single digital audio traffic stream, as well as the playout delay for the corresponding receiver over a 60-second simulation interval, for packet sizes $L_{p}=294$ and 882 bytes and for medium interference channel conditions. For these results, the SiS scheduler is employed, while the effect of the CoDeS synchronization algorithm to the playout delay is shown in the lower diagrams. Figure 7 shows the corresponding Tx and $\mathrm{Rx}$ buffer usage and playout delay for $L_{p}=294$ and 882 bytes, when the SETT-EDD scheduler is employed, under similar medium interference channel conditions.

Provided that the total Tx and Rx buffer lengths for each audio traffic flow were selected equal to 5 Kbytes and $5 \mathrm{~K}$ 


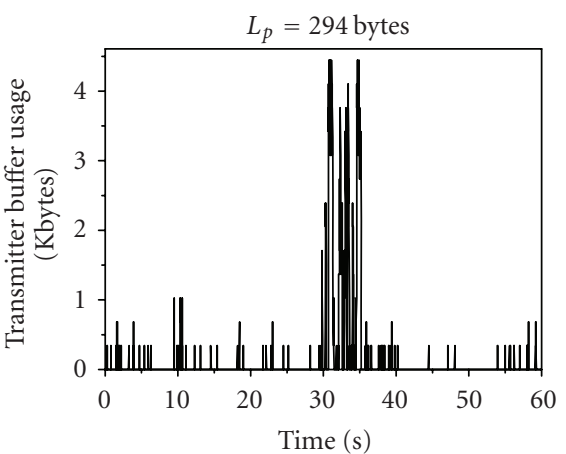

(a)

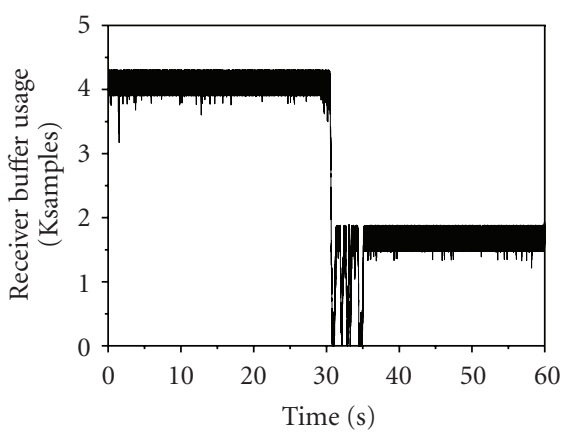

(c)

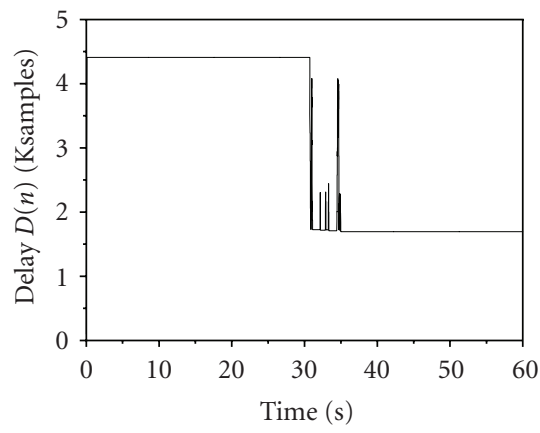

(e)

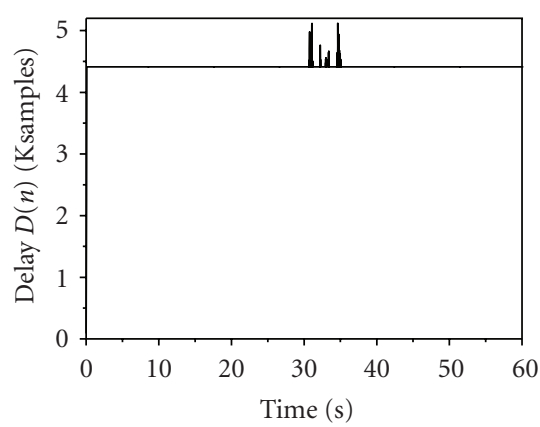

(g)

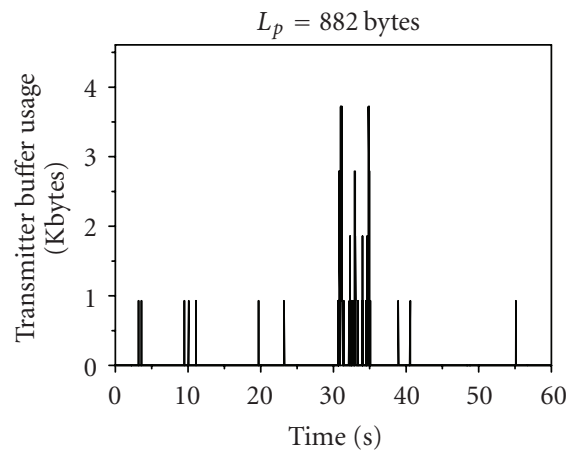

(b)

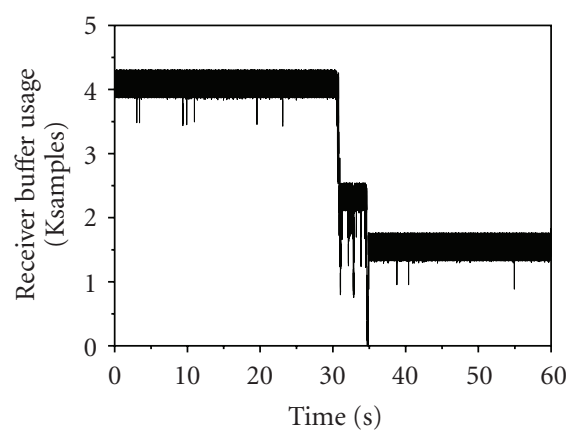

(d)

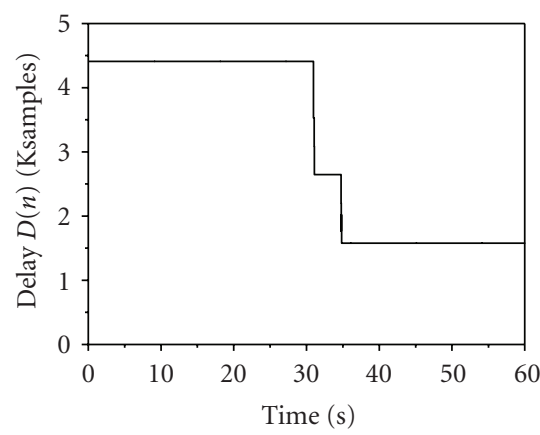

(f)

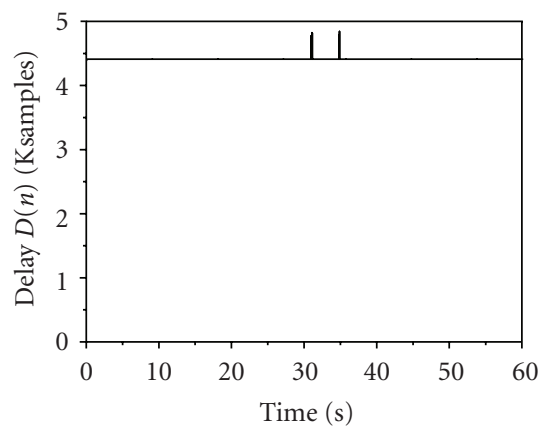

(h)

Figure 6: Typical example for time evolution in Tx and Rx buffer and end-to-end delay. One channel of a stereo setup is shown for different packet sizes (for SiS scheduler and medium interference): (a), (b) Tx buffer usage; (c), (d) Rx buffer usage; (e), (f) delay $D(n)$ without CoDeS synchronization; $(\mathrm{g})$, (h) delay $D(n)$ with CoDeS synchronization. 


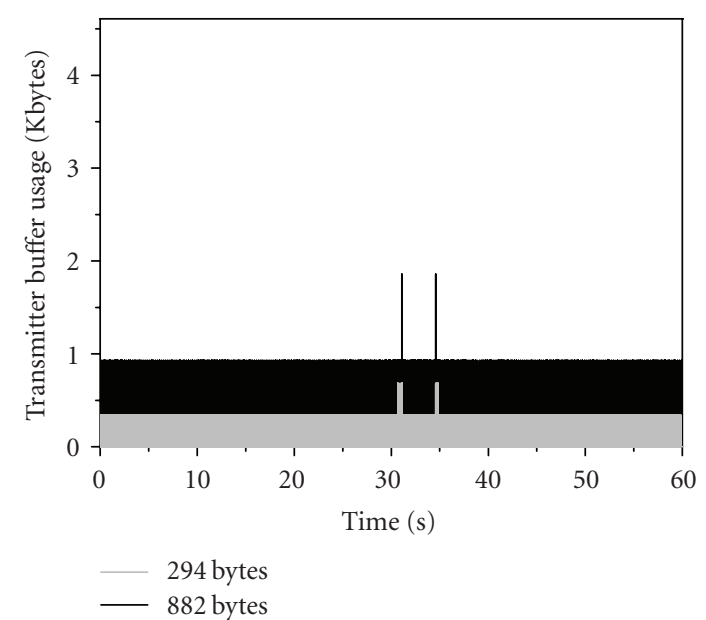

(a)

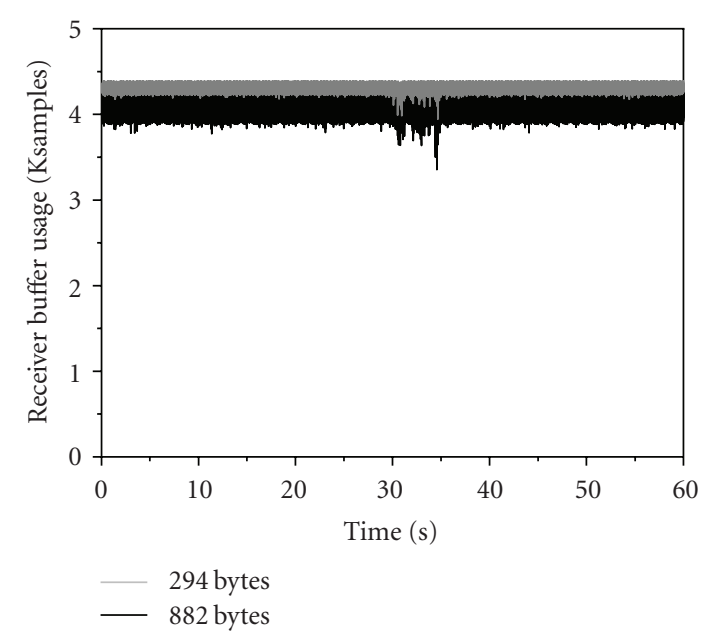

(b)

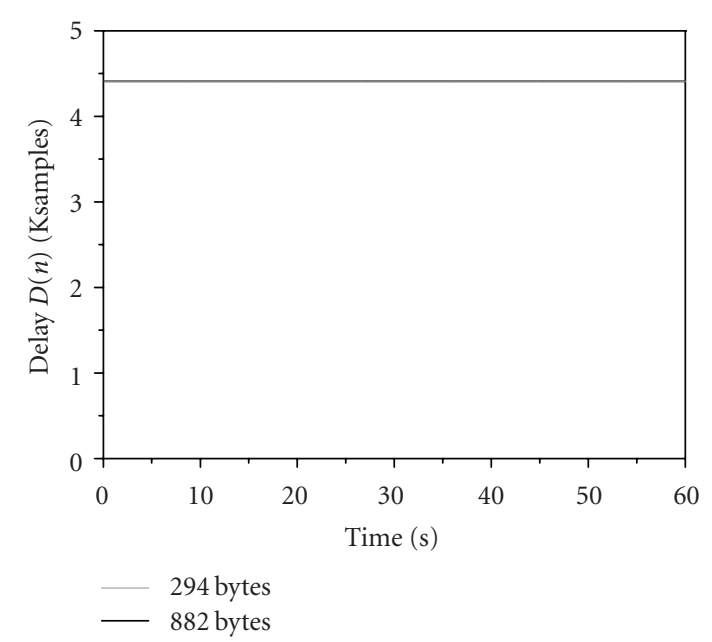

(c)

Figure 7: Typical example for time evolution in Tx and Rx buffer and end-to-end delay. One channel of a stereo setup is shown for different packet sizes (SETT-EDD scheduler and medium interference): (a) Tx buffer usage, (b) Rx buffer usage, (c) delay $D(n)$ without and with CoDeS synchronization (identical lines). samples, respectively; the maximum data that can be inserted into the corresponding queue is equal to the nearest, lowest multiple integer of the packet size employed. It should be also noted that when the playback starts, the initial Rx queue filling size equals to 4410 samples, due to the $100 \mathrm{~ms}$ prebuffering applied.

From the above tests, the following conclusions can be drawn.

(a) The SiS scheduler introduces overflows in the $\mathrm{Tx}$ buffer and significant data losses. Accordingly, the Rx buffer remains empty of data at many instances during the same interval, causing gaps in reproduction.

(b) For the case of the SETT-EDD, the minimum $\mathrm{Rx}$ buffer usage value for all tests is nearly 2500 samples for the largest packet considered. Thus, the prebuffering time can be reduced, without generating gaps.

(c) The Tx buffer is optimally utilized when using the SETT-EDD scheduler. For medium wireless channel conditions, higher buffer usage will be required; however, no overflows have occurred during the tested interval. It can be deduced that even for a smaller Tx buffer, the system would operate without data losses.

(d) Although the SiS scheduler performance is poor for all test cases, less erratic - but still not acceptable-playback is performed for $L_{p}=882$ bytes. On the other hand, no playback distortions occur when using the SETT-EDD scheduler, while improved operation is achieved for smaller packet sizes.

(e) The proposed application-level CoDeS synchronization algorithm generally ensures that the reproduction is kept synchronized for all test cases, since the delay for each traffic stream is constant throughout the simulation time.

The effect of the data overflows in the TxQ and the wireless variable packet delay transmission are clearly shown in Figure 8, where the original transmitted and the wirelessly reproduced waveforms are shown for a single audio channel. Apart of the silence gaps, a significant shift of the original waveform to the right side of the plot diagram is observed, which introduces relative channel phase delay. The audibility of both types of distortions introduced (silence gaps and relative channel delay) was verified through a sequence of tests, analyzed in the following section.

\subsubsection{Audibility of distortions}

Over the past years a number of psychoacoustic models and methods had been proposed to measure the perceived quality of both speech and audio signals [28]. The emergence of these approaches formulated up, to a certain degree, the ITU-R recommendation on perceptual evaluation of audio quality (PEAQ) [28, 29]. In the present study, two different methods were employed to assess the audibility of the wireless network distortions and to evaluate the performance of the interstream synchronization algorithm, on both PCM and compressed data: (a) the well-accepted noise-to-mask (NMR) criterion [30] and (b) a number of subjective listening tests.

Although the NMR criterion was initially developed for the purposes of perceptual audio coding, it can be also 


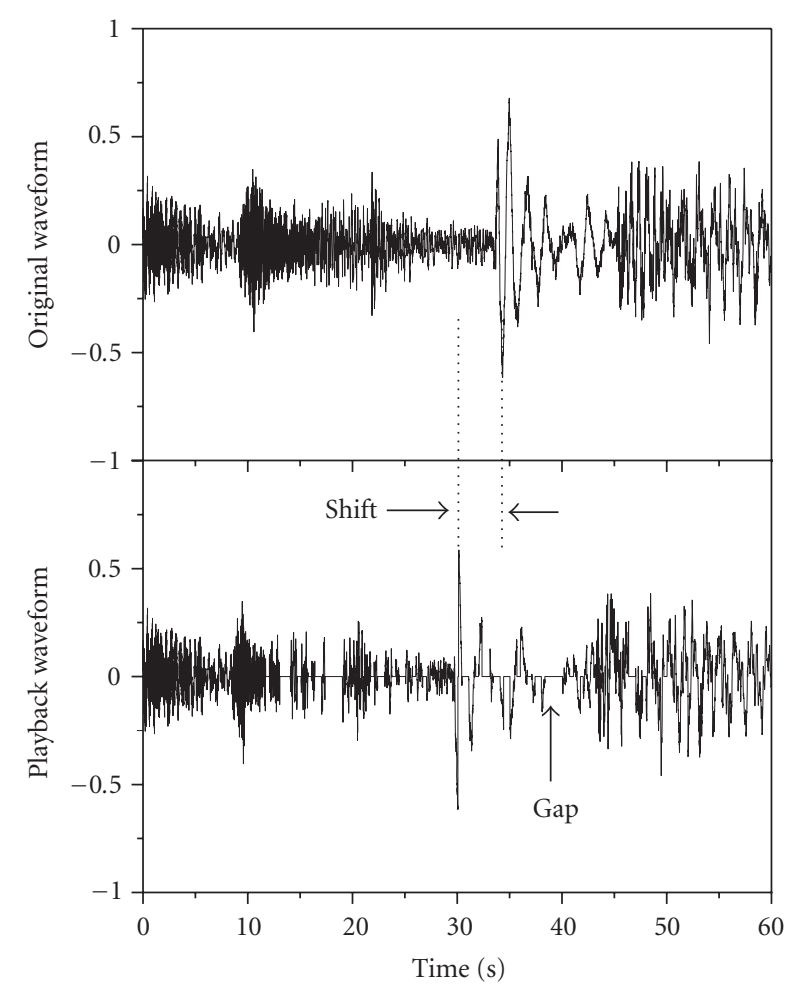

Figure 8: (a) Original digital audio source waveform, (b) reproduced (playback) waveform.

utilized to any audio processing system [31]. For NMR estimation $(\mathrm{dB})$ in frame $i$, the ratio between the "error" energy and masked threshold, grouped in a number of critical bands, was calculated in a frame by frame basis, that is,

$$
\operatorname{NMR}(i)=10 \cdot \log _{10}\left(\frac{1}{27} \sum_{\mathrm{cb}=1}^{27} \frac{\operatorname{error}_{\mathrm{cb}}(i)}{\text { mask_thr }_{\mathrm{cb}}(i)}\right)
$$

The objective metric utilized for the quality assessment of audio streams was based on the averaged $\operatorname{NMR}(i)$ values, for a total number of $\mathrm{K}$ frames. It should be noted that NMR values above $0 \mathrm{~dB}$ indicate the presence of audible distortions, while NMR values below $10 \mathrm{~dB}$ indicate an audio signal free of audible distortions [30] and that the reference signal used in all test cases was the original PCM audio track (prior to wireless transmission and any encoding/decoding).

The subjective listening tests considered both raw PCM and MPEG-1 Layer III audio streams, both wireless channel conditions (no and medium interference), as well as the proposed CoDeS synchronization algorithm. The tests were organized as follows: a total of 9 listeners participated in two successive sessions where identical test files were reproduced randomly in two phases. In phase $A$, the uncompressed audio signals were presented to the subjects and in phase $B$, the encoded/decoded audio test signals were presented. For both cases, the listeners were informed about the type of signals being reproduced, thus, to consider inherent degradation of the encoded/decoded audio signals. The listeners ranked the quality of the audio material in the scale from 1 to 5 where
1 was described as "bad," 2 as "poor," 3 as "fair," 4 as "good," and 5 as "excellent."

Figures 9 and 10 show the average NMR values, while Figures 11 and 12 show the corresponding subjective listening results as a function of the test parameters, for the case of raw PCM and mp3-coded audio.

From these figures, the following conclusions can be drawn.

(a) For raw PCM audio streams, the effect of the wireless network conditions in most cases is inaudible. However, using the SiS scheduler under medium interference wireless channel conditions (for both choices of packet lengths) introduces a notable audible degradation.

(b) This degradation is reduced when the proposed CoDeS synchronization algorithm is employed, which compensates for such distortions and reduces the average NMR values by $23 \mathrm{~dB}$ (for 294 bytes packet length) and $34 \mathrm{~dB}$ (for 882 bytes packet length). This can be also observed at Figure 11, where the CoDeS synchronization algorithm was ranked with higher score values.

(c) For compressed audio streams, it is clear that the inherent distortions due to the lossy data compression bias the NMR measurements, as it is also depicted in Figure 12 where generally lower score values are observed. However, it is clear that the wireless network imposes additional significant degradation to the overall audio quality, for almost all cases of using the SiS scheduler, especially during medium interference wireless channel conditions.

(d) For a coding rate of $256 \mathrm{kbps}$ and even for good channel conditions, the SiS scheduler introduces significant quality degradations, which again are reduced by the CoDeS synchronization algorithm (see Figures 10(b) and 12(b)). In these cases, the channel bandwidth usage is suboptimal due to the packet size employed (equal to the mp3 frame length).

(e) In Figure 10(b), NMR values for three of the test cases (i.e., $160 \mathrm{kbps}$ bitrate) are close to $10 \mathrm{~dB}$, which ideally indicate an audio signal free of audible distortions. However, Figure 12(b) indicates audio quality below "fair" for the test cases considered. As it is clear, full compliance between the subjective and objective tests is difficult to succeed, that is, instant audible distortions may cause the listener(s) to rank the entire audio segment as "fair" or even "poor." According to this approach, it is possible to have a biased subjective ranking (towards to low-grade audio quality) even for the highest bitrate (i.e., $256 \mathrm{kbps}$ ).

(f) Using the CoDeS synchronization algorithm in compressed audio (mp3) streams, an overall perceptual improvement equal to $7 \mathrm{~dB}$ can be achieved. More specifically, the distortions for the SiS scheduler, under medium interference wireless channel conditions, are compensated for both bitrates considered here (i.e., $160 \mathrm{kbps}$ and $256 \mathrm{kbps}$ ). This is also stated in Figure 12(b) as the score values for the test cases considered are above 2 indicating a slightly better perceived audio quality.

(g) As expected, the adaptive nature of the SETT-EDD scheduler leads to an overall better performance compared to the SiS scheduler, for all channel configurations and audio material. 


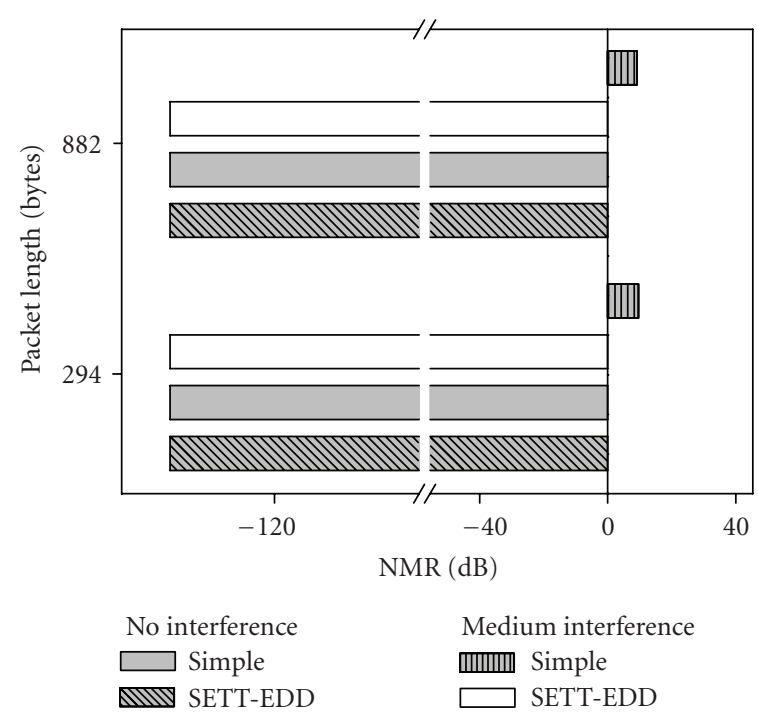

(a)

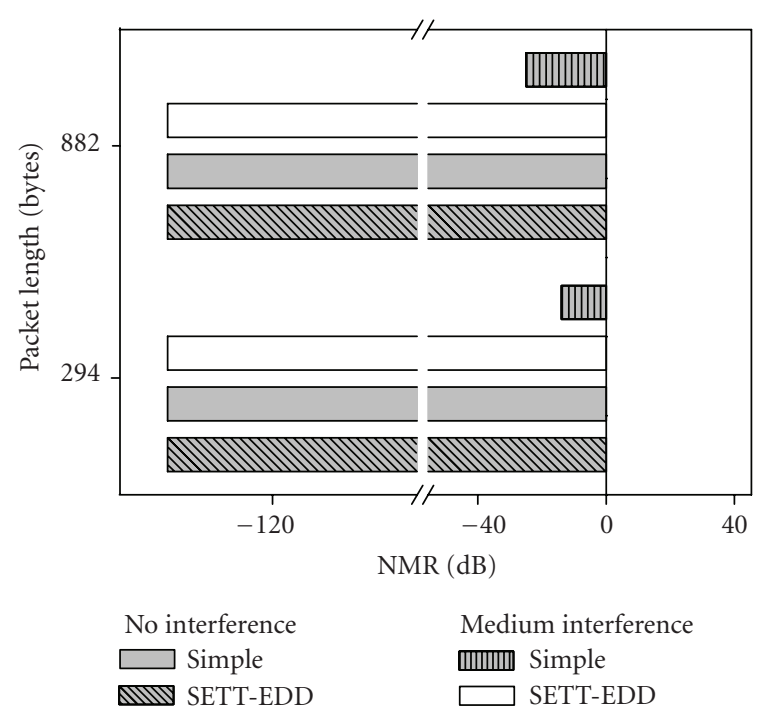

(b)

FIgUre 9: NMR values for 1-channel PCM audio WLAN streaming delivery: (a) CoDeS disabled; (b) CoDeS enabled.

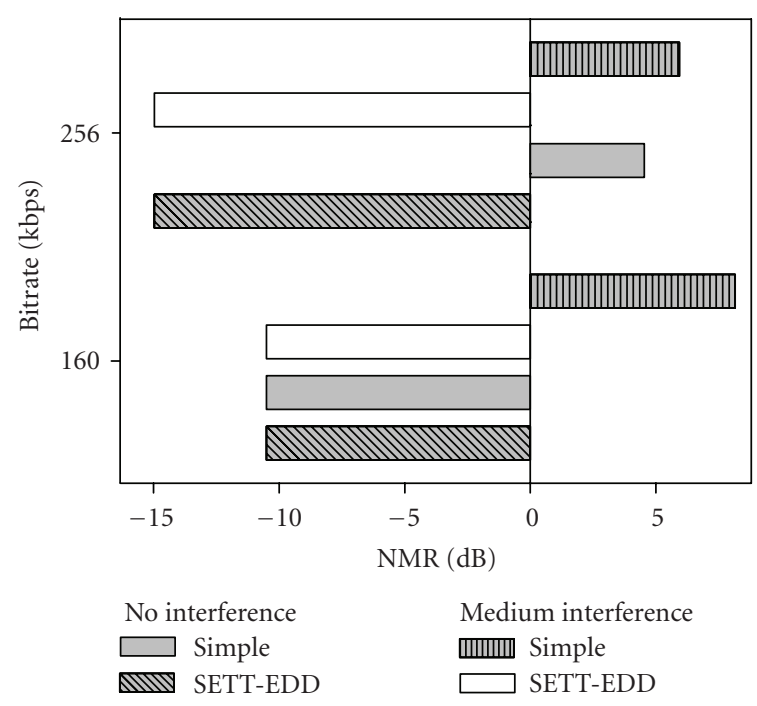

(a)

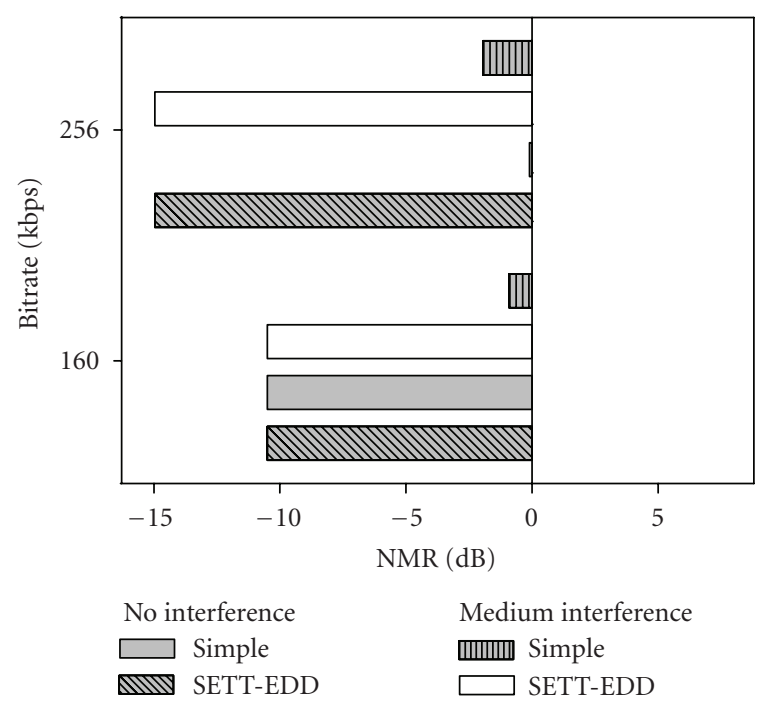

(b)

FIGURe 10: NMR values for MPEG-1 Layer III audio streaming delivery: (a) CoDeS disabled; (b) CoDeS enabled.

(h) Clearly, the results of the listening tests are in close agreement with the audio quality assessment performed using the NMR criterion.

Summarizing the above results, it is obvious that the wireless channel has a significant impact on the overall perceived playback audio performance. Nevertheless, from a networking point of view, the choice of the scheduler represents a critical decision, as it can render the presence of any wireless channel interference, transparent to the application. Moreover, it is clear that the proposed CoDeS synchronization strategy significantly improves the playback quality of both compressed and uncompressed audio for the case of low wireless link quality.

\subsubsection{Overall audio WLAN performance}

Error-free playback can be achieved when the output audio streams match sample-accurately the input streams; after taking out the initial delay caused by the prebuffering stage, Figure 13 shows the error-free stereo PCM and mp3 playback test cases examined under good and medium wireless channel conditions. The above methodology was extended to a wireless 5-channel PCM (16 bit/44.1 KHz) playback system and the number of error-free reproduced audio channels for this case is shown in Figure 14. It can be deduced that when interference is present, smaller packet lengths and the employment of an adaptive scheduler (such as the SETT-EDD) should be preferred. 


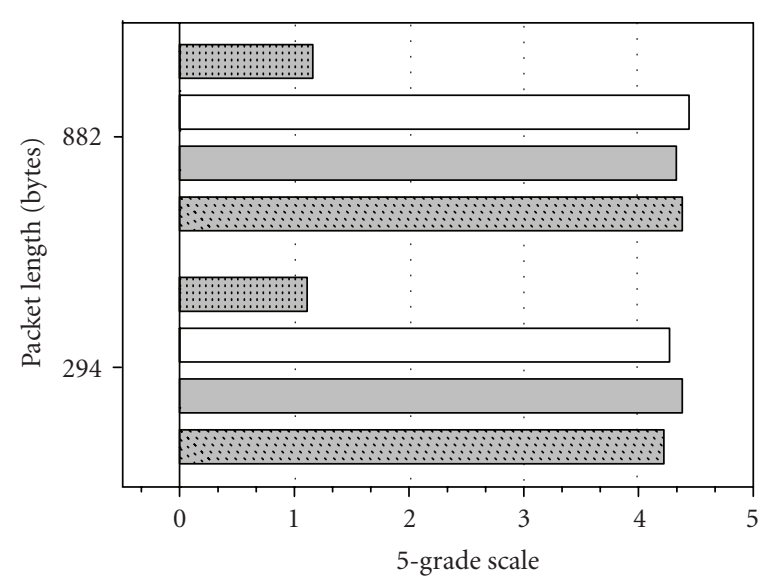

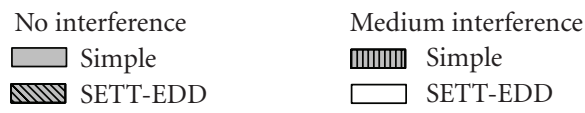

(a)

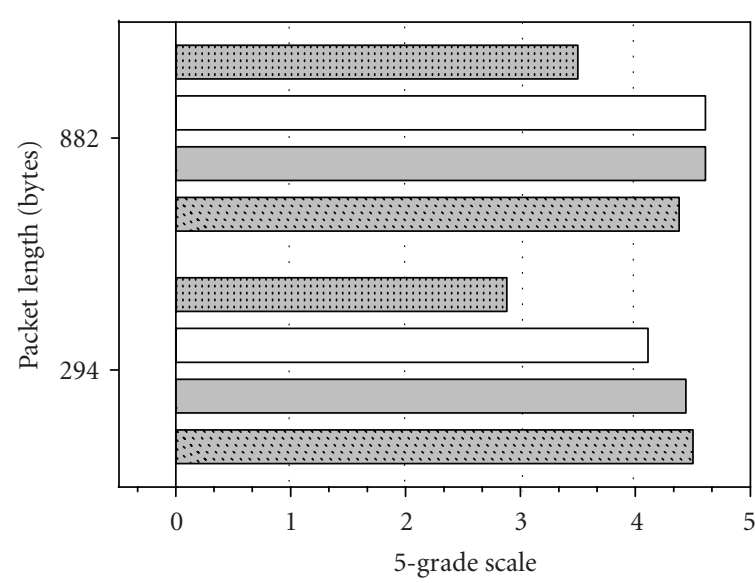

No interference Medium interference

$\square$ Simple $\quad$ 盂盂 Simple

MIV SETT-EDD

(b)

FIGURE 11: Subjective test results for PCM audio WLAN streaming delivery: (a) CoDeS disabled; (b) CoDeS enabled.

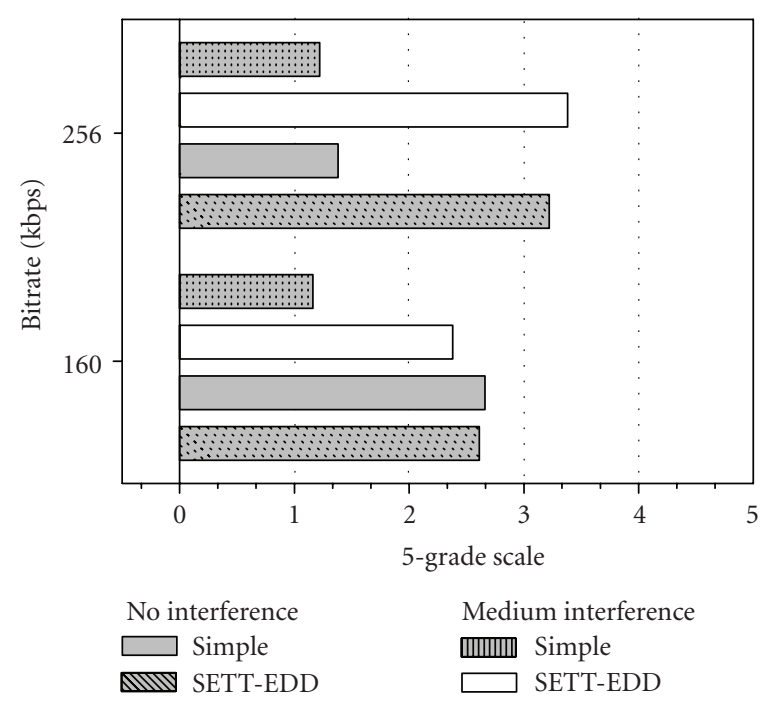

(a)

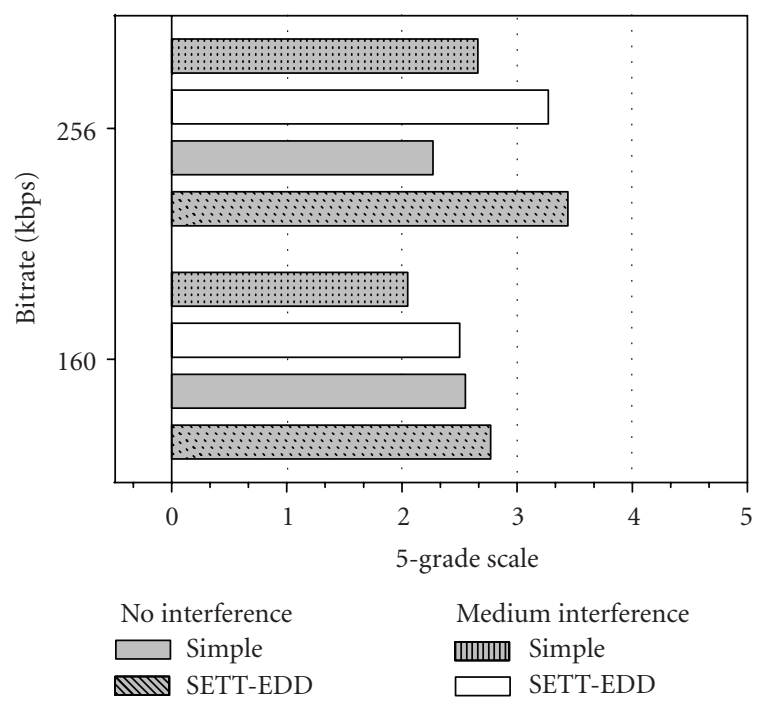

(b)

FIgURE 12: Subjective test results for MPEG-1 Layer III audio streaming delivery: (a) CoDeS disabled; (b) CoDeS enabled.

Finally, Figure 15 shows the measured delayed throughput $\left(T_{D}\right)$ values (see $\left.(13)\right)$ for the wireless transmission of stereo mp3, stereo PCM $16 \mathrm{bit} / 44.1 \mathrm{KHz}, 5$-channel PCM $16 \mathrm{bit} / 44.1 \mathrm{KHz}$, and stereo PCM $24 \mathrm{bit} / 96 \mathrm{KHz}$ audio, using the simple and the SETT-EDD scheduler, under medium interference wireless channel conditions. As can be deduced from (13), $T_{D}$ (Mbps) indicates the mismatch between the requested (from the audio source) and the on-time WLAN delivered data. Note that for mp3 and stereo CD-quality transmission, the delayed throughput for SETT-EDD is zero. It appears that for noncompressed PCM audio and for in- creasing source bitrate, a corresponding increase of $T_{D}$ can be expected. However, for mp3 compressed audio transmission, due to the additional overhead caused by the mismatch between the $\mathrm{mp} 3$ frame length and the network packet length, a strong increase of the measured $T_{D}$ values must be expected.

Clearly, while the packet length does not seem to have a direct impact on the playback performance for PCM audio transmission, smaller packet sizes are more robust, since the Tx and Rx buffers are optimally used. The test cases prove that the selection of the service scheduler, especially 


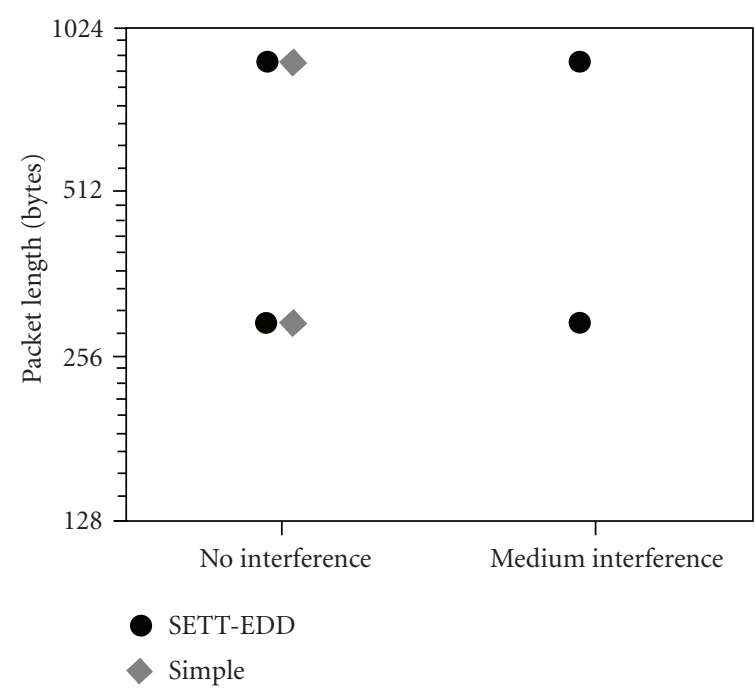

(a)

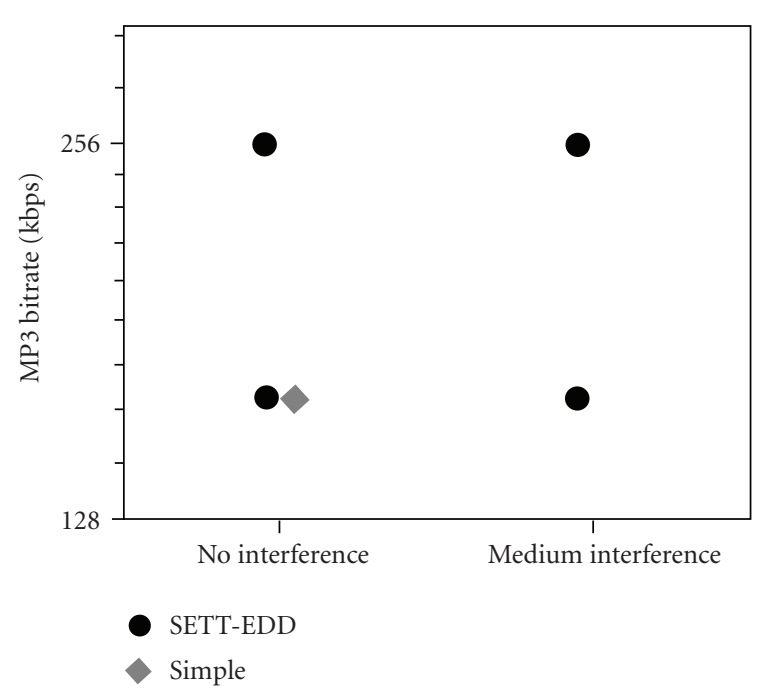

(b)

FIGURE 13: Parameter map for error-free stereo digital audio WLAN delivery: (a) PCM $f_{s}=44.1 \mathrm{KHz}, N=16$ bit; (b) mp3 coded at $160 \mathrm{Kbps}$ and 256 Kbps.

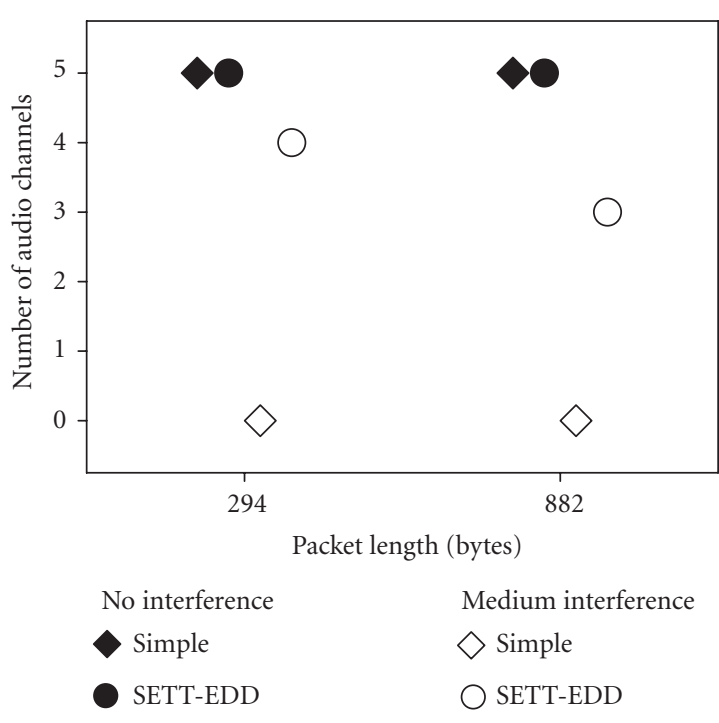

FIGURE 14: Number of serviced audio channels versus packet length for error-free digital WLAN delivery.

under the presence of channel interference, as in probable real-life conditions plays a crucial role on the overall playback performance. More specifically, it was found that the simple scheduler defined by the 802.11e specification as a minimum requirement reference design introduces significant reproduction distortions for most test cases, even for mp3 transmission under medium channel interference. Note that the simple scheduler induces distortions in all receivers of a 5-channel PCM system and completely fails to service high quality $96 \mathrm{KHz} / 24$ bit PCM stereo.
On the other hand, largely error-free reproduction is achieved for the case of the SETT-EDD scheduler, although some distortions can be expected for the cases of 5-channel PCM and $96 \mathrm{KHz} / 24$ bit stereo reproduction, largely due to the higher bitrate required. The trends obtained from the error-free results for the SETT-EDD scheduler would apply even if the Tx and $\mathrm{Rx}$ buffer lengths are reduced and starter prebuffering time is applied. This can enhance the overall performance of the WLAN digital audio system, especially for applications where a maximum delay limit is imposed, such as for typical audiovisual applications.

\section{CONCLUSIONS}

The most challenging aspect of integrating digital audio and WLAN technologies appears to be the robust and synchronized real-time streaming of multiple channels to multipoint receivers. Although efficient, cost-effective, and wellestablished network topologies exist for such applications, it appears that these solutions are not yet transparent for audio applications. An initial conclusion derived from this study is that successful operation can be achieved only if the wireless protocol provides strict QoS guarantees.

Main sources of distortion are due to audio WLAN packets, being permanently lost, generating gaps and loss of synchronization between the reproduced audio channels. The results presented in the previous section describe the optimal parameters for error-free stereo CD-quality and $\mathrm{mp} 3$ real-time audio playback. While it may be assumed that the 802.11b 11 Mbps throughput should be sufficient for uncorrupted $\mathrm{mp} 3$ and PCM stereo playback, such comparisons between the audio source bitrate and the physical channel throughput do not suffice for concluding that the application 


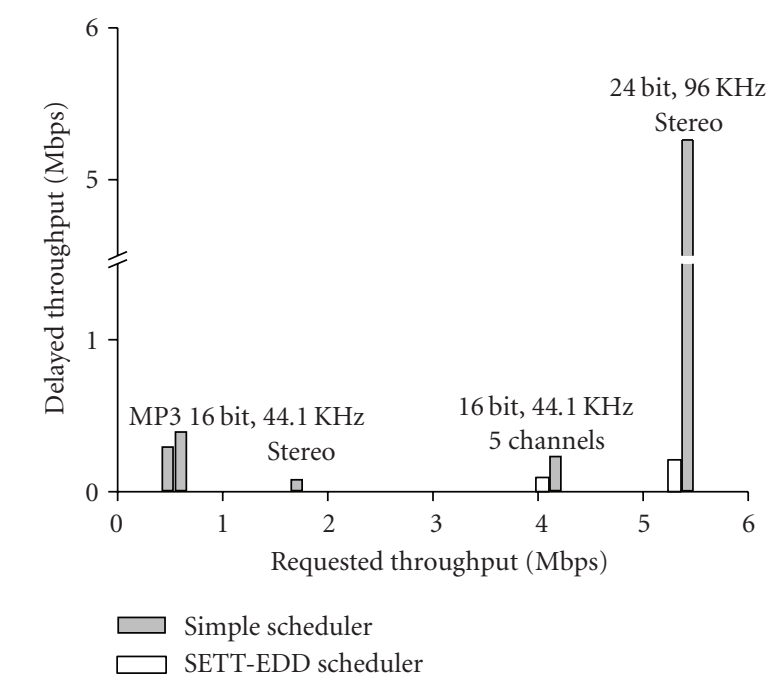

FIGURE 15: Delayed throughput for digital WLAN audio delivery.

will not suffer from such WLAN distortions. The largely error-free reproduction achieved for the case of the SETTEDD scheduler shows that the employment of an adaptive scheduler, which dynamically adjusts the service schedule based on the networking conditions, represents a fundamental requirement for practical real-time audio streaming applications, at the expense of increased implementation complexity and processing power. However, excessive channel interference may always introduce additional transmission errors and channel congestion. Under such conditions and due to the wireless network bandwidth limitations, any dynamic scheduling algorithm (such as the SETT-EDD scheduler) may also fail to service real-time streaming traffic flows.

An application-level mechanism such as the proposed CoDeS synchronization algorithm will generally ensure that the reproduction is kept synchronized for WLAN transmission-induced errors by keeping the delay for each traffic stream constant, for each serviced device. Additionally, although it is out of the scope of this work, it is expected that the CoDeS synchronization algorithm combined with a packet concealment method $[32,33]$ will minimize the perceptual effect of the distortions that are introduced by the insertion of silence gaps and proportionally increase the achieved playback quality. Furthermore, by increasing the available bandwidth, the effect of the channel interference on the final playback quality can be reduced. Hence, highrate wireless protocols (e.g., $801.11 \mathrm{~g} / \mathrm{n}$ ) must be preferred for high-quality multimedia and audio applications. Additionally, higher-layer protocols can be developed for dynamically adjusting the number of the playback devices which can be serviced within such a WLAN. For example, under wireless channel degradation, such protocols may temporarily stop servicing playback devices with lower priority on the perceived audio system quality (e.g., the rear speakers in a multichannel DVD setup).

\section{ABBREVIATIONS}

AP:

CoDes:

EDCA:

FIFO:

HCCA:

ISM:

LAN:

MAC:

PHY:

QAP:

QoS:

$\mathrm{Rx}$ :

Access point

Consistent delay synchronization

Enhanced distributed channel access

First-in first-out

Hybrid controlled channel access

Industrial, scientific, medicine

Local area network

Medium access control

Physical rate

Quality of service access point

Quality of service

SETT-EDD: Scheduling based on estimated transmission times-earliest due date

SiS: $\quad$ Simple scheduler

STA: Wireless station

TSPEC: Traffic specification

Tx: Transmission

TXOP: $\quad$ Transmission opportunity

WiDAR: Wireless digital audio receiver

WiDAS: Wireless digital audio source

WLAN: Wireless local area network.

\section{REFERENCES}

[1] T. Blank, B. Atkinson, M. Isard, J. D. Johnston, and K. Olynyk, "An internet protocol (IP) sound system," in Proceedings of the 117th Convention of the Audio Engineering Society, San Francisco, Calif, USA, October 2004, (preprint 6211).

[2] Bluetooth SIG, "Specification of the bluetooth system," bluetooth core specification version 2.0 EDR [ vol 0], November 2004.

[3] European Telecommunications Standards Institute (ETSI), "Broadband radio access networks (BRAN)," HIPERLAN Type 2 Specification.

[4] “The HomeRF ${ }^{\mathrm{TM}}$ Technical Committee," HomeRF Specification, Revision 2.01, July 2002.

[5] IEEE802.11 WG and IEEE802.11, "Information technology telecommunications and information exchange between system local and metropolitan area networks-specific requirements-part 11: wireless LAN medium access control (MAC) and physical layer (PHY) specifications: higherspeed physical layer extension in the $2.4 \mathrm{GHz}$ band," September 1999.

[6] IEEE802.11 WG and IEEE802.11g, "IEEE standard for information technology-telecommunications and information exchange between systems-local and metropolitan area networks-specific requirements-part 11: wireless LAN medium access control (MAC) and physical layer (PHY) specifications amendment 4: further higher data rate extension in the 2.4 GHz band," June 2003.

[7] N.-A. Tatlas, A. Floros, and J. Mourjopoulos, "Wireless digital audio delivery analysis and evaluation," in Proceedings of the 31th IEEE International Conference on Acoustics, Speech and Signal Processing (ICASSP '06), vol. 5, pp. V201-V204, Toulouse, France, May 2006.

[8] H. Liu and M. Zarki, "A synchronization control scheme for real-time streaming multimedia applications," in Proceedings of the 13th IEEE International Packet Video Workshop, Nantes, France, April 2003. 
[9] P. Blum and L. Thiele, "Trace-based evaluation of clock synchronization algorithms for wireless loudspeakers," in Proceedings of the 2nd Workshop on Embedded Systems for RealTime Multimedia (ESTIMedia '04), pp. 7-12, Stockholm, Sweden, September 2004.

[10] A. Xu, W. Woszczyk, Z. Settel, et al., "Real-time streaming of multichannel audio data over Internet," Journal of the Audio Engineering Society, vol. 48, no. 7, pp. 627-639, 2000.

[11] IEEE802.11 WG and IEEE802.11e/D13.0, "IEEE standard for information technology-telecommunications and information exchange between systems-local and metropolitan area networks-specific requirements-part 11: wireless medium access control (MAC) and physical layer (PHY) specifications: amendment: medium access control (MAC) quality of service enhancements," January 2005.

[12] A. Floros, N.-A. Tatlas, and J. Mourjopoulos, "BlueBox: a cable-free digital jukebox for compressed-quality audio delivery," IEEE Transactions on Consumer Electronics, vol. 51, no. 2, pp. 534-539, 2005.

[13] http://www.streamium.com/.

[14] http://www.elevenengineering.com/.

[15] http://www.amphony.com/products/h2500.htm.

[16] http://www.pioneerelectronics.com/.

[17] N.-A. Tatlas, A. Floros, P. Hatziantoniou, and J. Mourjopoulos, "Towards the all-digital audio/acoustic chain: challenges and solutions," in Proceedings of the AES 23rd International Conference on Signal Processing in Audio Recording and Reproduction, Copenhagen, Denmark, May 2003.

[18] J. S. Flaks, "Quality of service (QoS) for streaming audio over wireless LANs," in Proceedings of the AES 18th International Conference: Audio for Information Appliances, Burlingame, Calif, USA, March 2001.

[19] A. Floros and T. Karoubalis, "Delivering high-quality audio over WLANs," in Proceedings of the 116th AES Convention of the Audio Engineering Society, Berlin, Germany, May 2004, (preprint 5996).

[20] A. Floros, T. Karoubalis, and S. Koutroubinas, "Bringing quality in the 802.11 wireless arena," in Broadband Wireless and WiMax IEC Comprehensive Report, International Engineering Consortium, Chicago, Ill, USA, 2005.

[21] S. Mangold, S. Choi, G. R. Hiertz, O. Klein, and B. Walke, "Analysis of IEEE 802.11 e for QoS support in wireless LANs," IEEE Wireless Communications, vol. 10, no. 6, pp. 40-50, 2003.

[22] A. Grilo, M. Macedo, and M. Nunes, "A scheduling algorithm for QoS support in IEEE802.11E networks," IEEE Wireless Communications, vol. 10, no. 3, pp. 36-43, 2003.

[23] X. Gu, M. Dick, Z. Kurtisi, U. Noyer, and L. Wolf, "Networkcentric music performance: practice and experiments," IEEE Communications Magazine, vol. 43, no. 6, pp. 86-93, 2005.

[24] N.-A. Tatlas, A. Floros, T. Zarouchas, and J. Mourjopoulos, "An error-concealment technique for wireless digital audio delivery," in Proceedings of the 5th International Conference on Communication Systems, Networks and Digital Signal Processing (CSNDSP '06), pp. 181-184, Patras, Greece, July 2006.

[25] H. Ofir and D. Malah, "Packet loss concealment for audio streaming based on the GAPES algorithm," in Proceedings of the 118th Convention of the Audio Engineering Society, Barcelona, Spain, May 2005, (preprint 6334).

[26] N.-A. Tatlas, A. Floros, and J. Mourjopoulos, "An evaluation tool for wireless digital audio applications," in Proceedings of the 118th Convention of the Audio Engineering Society, Barcelona, Spain, May 2005, (preprint 6386).

[27] A. K. Salkintzis, G. Dimitriadis, D. Skyrianoglou, N. Passas, and N. Pavlidou, "Seamless continuity of real-time video across UMTS and WLAN networks: challenges and performance evaluation," IEEE Wireless Communications, vol. 12, no. 3, pp. 8-18, 2005.

[28] C. Colomes, C. Schmidmer, T. Thiede, and W. C. Treurniet, "Perceptual quality assessment for digital audio: PEAQ- the New ITU standard for objective measurement of the perceived audio quality," in Proceedings of the 17th International Conference on the Audio Engineering Society (AES '99), Florence, Italy, September 1999.

[29] S. Bech and N. Zacharov, Perceptual Audio EvaluationTheory, Method and Application, John Wiley \& Sons, New York, NY, USA, 2006.

[30] K. Brandenburg and T. Sporer, "NMR and masking flag: evaluation of quality using perceptual criteria," in Proceedings of the 11th International AES Conference: Audio Test \& Measurement, pp. 169-179, Portland, Oregon, May 1992.

[31] J. Herre, E. Eberlein, H. Schott, and K. Brandeburg, "Advanced audio measurement system using psychoacoustic properties," in Proceedings of the 92nd AES Convention of the Audio Engineering Society, New York, NY, USA, March 1992, (preprint 3321).

[32] B. W. Wah, S. Xiao, and L. A. Dong, "A survey of errorconcealment schemes for real-time audio and video transmissions over the Internet," in Proceedings of International Symposium on Multimedia Software Engineering, pp. 17-24, Taipei, Taiwan, December 2000.

[33] A. Floros, M. Avlonitis, and P. Vlamos, "Stochastic packet reconstruction for subjectively improved audio delivery over WLANs," in Proceedings of the 3rd International Mobile Multimedia Communications Conference (MOBIMEDIA '07), Nafpaktos, Greece, August 2007. 

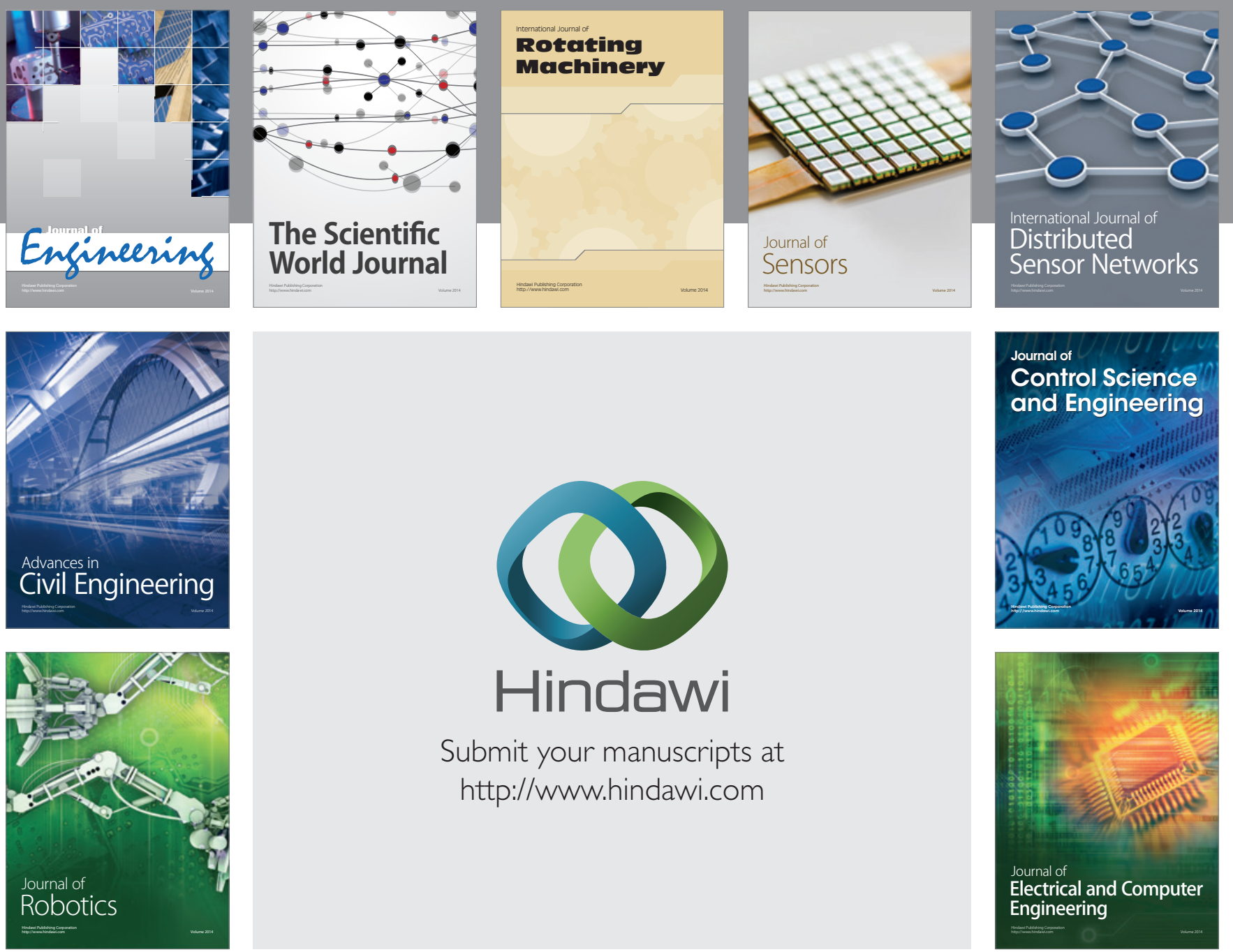

Submit your manuscripts at

http://www.hindawi.com
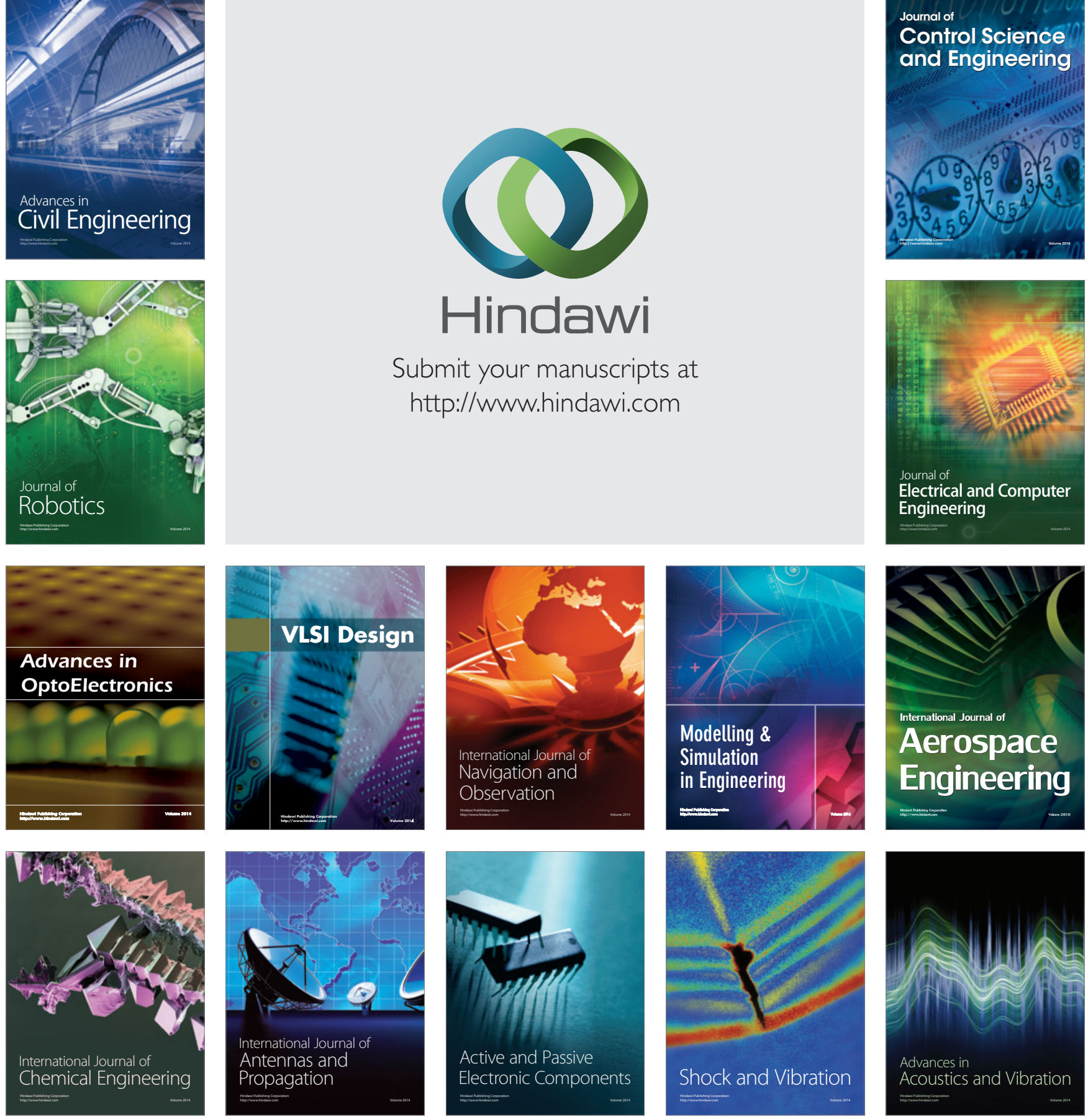